\title{
Riesgo psicosocial: tendencias y nuevas orientaciones laborales
}

\section{Psychosocial risk: trends and new labour guidelines}

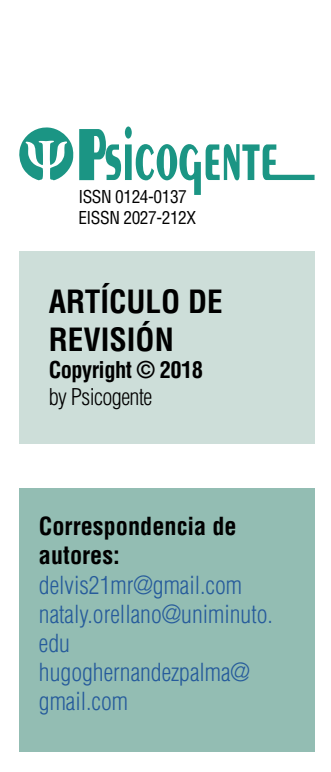

Recibido: $13-01-17$ Aceptado: $22-01-17$ Publicado: $01-07-18$

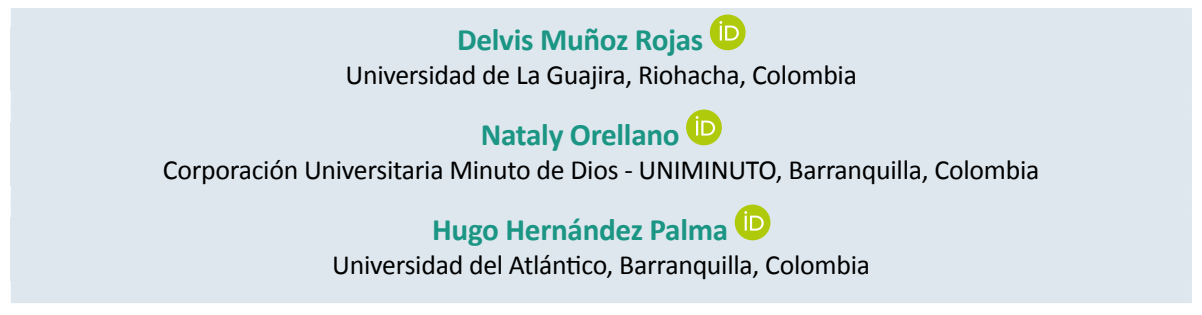

Resumen

Con el presente artículo de revisión se pretende identificar las tendencias y nuevas orientaciones laborales sobre el riesgo psicosocial en los diferentes sectores empresariales de Colombia, teniendo en cuenta los diversos factores presentes en los escenarios de ejercicio profesional de los trabajadores. En los últimos años, se ha observado un creciente impacto de las consecuencias de la exposición a los diferentes riesgos psicosociales dentro de las organizaciones, por lo cual resulta indispensable estudiar la normatividad aplicada en el entorno nacional e internacional, para clarificar la perspectiva que están manejando actualmente las organizaciones nacionales frente a estos riesgos, y si las medidas tomadas están siendo efectivas para la prevención de las consecuencias derivadas de la exposición del trabajador a los peligros propios de los distintos ambientes.

\section{Palabras clave: estrés, factores de riesgo, impacto, salud laboral, cultura organizacional.}

\section{Abstract}

This review paper aims to analyze and identify the trends and new labor guidelines related to psychosocial risk given in different business fields from our country and also, how these labor orientations take strength for Colombian workers, taking into account several factors into different scenarios. A growing impact of psychosocial risk consequences within organizations, has been observed in recent years; That is why, it becomes essential to study the normativity applied in the national and international context, in order to clarify the current point of view related to how national organizations deal with these kind of risks, and whether the measures taken are really effective in preventing the consequences of workers' exposure to this type of hazard inherent in all working environments.

Key words: stress, risk factors, impact, occupational health and organizational culture.

Cómo citar este artículo (APA):

Muñoz Rojas, D., Orellano, N. \& Hernández Palma, H. (2018). Riesgo psicosocial: tendencias y nuevas orientaciones laborales. Psicogente 21(40), $532-544$. https://doi.org/10.17081/psico.21.40.3090 


\section{INTRODUCCIÓN}

Los diversos y constantes cambios de la economía, así como el progreso sostenido de la industria y de las diferentes organizaciones empresariales introducen variantes relevantes en el mundo laboral. Esto exige la realización de estudios enfocados en los factores de riesgos laborales y psicosociales inherentes a estos cambios, a fin de prevenir tales riesgos y favorecer la creación de entornos saludables teniendo en cuenta las interacciones laborales, las condiciones organizacionales y el entorno de desempeño. Así se puede garantizar la productividad y el crecimiento empresarial.

En la actualidad, los datos suministrados por la Organización Mundial de la Salud (OMS) evidencian que dos millones de hombres y mujeres mueren anualmente como resultado de accidentes, enfermedades o lesiones relacionadas con el trabajo. Se debe tener en cuenta, además, que existen accidentes de trabajo no fatales, los cuales provocan en promedio al menos tres días de ausentismo por incapacidad y 160 millones de casos nuevos por enfermedad laboral. Asimismo, se ha detectado que un $8 \%$ de la tasa global de trastornos depresivos en la actualidad, se relaciona con riesgos ocupacionales (OMS, 2010).

La Organización Internacional del Trabajo (OIT) y la OMS han venido abordando el tema de riesgo psicosocial desde la salud ocupacional, la psicología organizacional y el desarrollo humano, haciendo un importante énfasis en las relaciones sociales y laborales. Los empleados de una organización influyen de manera notoria en su ambiente. En este sentido, es imposible imaginar las organizaciones sin seres humanos, pues por más que la tecnología avance, el recurso humano es quien les da vida y humaniza la relación cliente-empresa. En este marco, es imposible obviar los factores psicosociales que intervienen en su ambiente laboral (Cox, 2004). Estos espacios se integran y promueven la creación de barreras protectoras que permiten enfrentarse a los cambios del ambiente y optimizar su calidad de vida.

Por todo lo anterior, como se define en el título de este texto, el riesgo psicosocial y las consecuencias derivadas constituyen un trascendental reto para las naciones, los ámbitos económicos, los trabajadores, los accionistas, los empleadores, los profesionales de la salud, entre otros (OMS, 2010).

Los factores psicosociales se determinan por la desigualdad en la exposición; se ha demostrado que los trabajadores que realizan tareas de ejecución tienen exposiciones psicosociales más desfavorables para la salud que 
quienes realizan tareas de diseño o de planificación. Además, se debe tener en cuenta que las mujeres tienen peores condiciones de trabajo en la mayoría de casos. En este último sentido, las principales desigualdades en las empresas se pueden identificar atendiendo a las exposiciones psicosociales por puesto de trabajo y sexo (OMS, 2010).

\subsection{Tendencias y orientaciones}

Se ha demostrado la predisposición a consecuencias inmediatas de los riesgos a los que los trabajadores están expuestos a corto, mediano y largo plazo, tales como: físicos, químicos, biológicos, entre otros. Lo anterior acarrea enfermedades y accidentes, algunas veces mortales y otras graves.

El riesgo laboral se hace mayor cuando su impacto recae directamente sobre la vida de los trabajadores. No obstante, a raíz de los cambios dentro de las organizaciones, se ha derivado un desplazamiento importante de estos hacia el sector terciario laboral, que ha cambiado el perfil de riesgos y ha aumentado las consecuencias de los de origen organizacional y psicosocial. Los estudios que se han venido realizando aportan datos según los cuales los riesgos a los que está expuesto un trabajador pueden ser inmediatos, pero sus secuelas aparecen a corto, mediano y largo plazo. Entre tales riesgos, aun cuando no se evidencie, el psicosocial debe tener el mismo tratamiento que el resto y debe contemplar las implicaciones para el individuo en todas sus dimensiones.

La importancia creciente de los factores psicosociales proviene de su extensión, especialmente debido al aumento de organizaciones en el sector servicios. Cabe aclarar que los riesgos laborales de origen organizacional y psicosocial no son exclusivos del sector servicios, pues están presentes en el resto de sectores, pero su presencia en este sector es claramente prevalente, teniendo en cuenta que en este sector se están viviendo transiciones organizacionales y que la creciente creación de nuevas empresas en el gremio, no representa propiamente estabilidad laboral debido a las pocas garantías que ofrece, en nuestro caso, el gobierno de Colombia a los pequeños y medianos empresarios en materia tributaria. Ello genera depresión económica y en estas circunstancias las inversiones no se dirigen hacia el área de Seguridad y Salud en el Trabajo (SST), como tampoco representa rentabilidad para que una empresa impulse las condiciones de un trabajador o genere empleo.

Para que las empresas comprendan la importancia de los cuidados que supone el Sistema de Gestión de Seguridad y Salud en el Trabajo (SGSST) 
para los empleados y para los recursos financieros de la empresa, se necesita realizar un cambio de fondo de la perspectiva de productividad y competitividad, pues anteriormente el factor humano era considerado un medio o un costo más de la empresa, es decir, se asumía al ser humano como insumo y no como el actor principal que maneja los recursos disponibles y que reacciona de acuerdo con los procesos psicológicos y psicosociales que experimenta al actuar en sociedad.

La productividad debe verse entonces como el resultado del rendimiento del talento humano, que rodeado de las condiciones y de las medidas básicas de seguridad y bienestar laboral en la organización, podrán ofrecer todos sus esfuerzos para producir bienes y servicios en forma eficiente, por lo que toda intervención para mejorar la productividad en la organización tiene su génesis en las personas (Singh, 1998; Cox \& Rial-González, 2000). Lo anterior confirma que el recurso humano debe ser el primer objetivo de los directivos en una organización.

Son muchos los factores internos y externos que afectan directa o indirectamente la productividad organizacional. Por lo tanto, es importante identificarlos y estudiar las medidas que permitan a la organización gestionarlos, adoptando estrategias de impacto que produzcan los resultados esperados. Más en concreto, hay factores sobre los cuales es necesario diseñar estrategias que permitan enfrentar las situaciones adversas y aprovechar las oportunidades, lo cual requiere de un permanente monitoreo y análisis de los cambios en el entorno. En pocas palabras, se hace necesario el análisis de estas situaciones bajo el parámetro de un mejoramiento continuo que permita no solo identificarlas sino generar un plan de acción, verificación y evaluación para establecer si las estrategias utilizadas están siendo las adecuadas o si se requiere mejorar o cambiarlas en alguna parte del proceso, de modo que se puedan lograr los objetivos planteados en la organización respecto a la eliminación y control de los riesgos existentes. Como ya se dijo, los factores psicosociales vienen siendo estudiados por varios expertos y por algunas organizaciones dentro de las cuales se han desarrollado modelos para su prevención y su intervención en la dinámica organizacional. Uno de estos es el Modelo de Factores Psicosociales propuestos por el Comité Mixto de la Oganización Internacional del Trabajo (OIT) y la Organización Mundial de la Salud (OMS) sobre Medicina del Trabajo (1984). Este modelo busca identificar y controlar los factores de riesgos psicosociales que resultan altamente nocivos en el desarrollo de la actividad laboral, por lo que en él se postula que la interacción entre los individuos y su medioambiente de trabajo se deter- 
minan por las condiciones de trabajo y por las capacidades y necesidades humanas. De esta manera, es claro que existen unos factores primordiales dentro de esta interacción: la tarea, el medioambiente físico y de trabajo, las prácticas administrativas y las condiciones de empleo en general: "Los valores y limitaciones humanas que determinan el éxito de la interacción están fundadas en las características generales psicológicas y biológicas del individuo, así como en las peculiaridades individuales y en el contexto social" (OIT/OMS, 1984). Es importante recordar que dentro de las organizaciones actuales todo es cambiante y que, a pesar de que la estructura laboral es compleja, hace parte de esos cambios que se promueven casi que a diario. El informe del año 2006 de la Conferencia Internacional del Trabajo identifica al respecto cuatro fuerzas principales que actúan como motores del cambio en el mundo del trabajo: 1) En primer lugar aparece el imperativo del desarrollo; es decir, nos encontramos ante un mundo en constante progreso y crecimiento, en el que muchos procesos se tornan obsoletos de manera rápida $y$, por ende, propician la creación acelerada de nuevos procedimientos; 2) En segundo lugar, se encuentra la transformación tecnológica como principal causa de los cambios mencionados antes, teniendo en cuenta que a mayor tecnología se percibe una menor necesidad del recurso humano lo que causa estrés en el trabajador ante la posible pérdida del puesto de trabajo; 3) La intensificación de la competencia a escala mundial hace mayor la exigencia en los procesos. 4) Por último, es importante la mayor orientación en función de los mercados y la función más reducida del Estado.

Vale la pena recordar que en las últimas décadas los riesgos psicosociales y sus consecuencias, han tomado gran importancia por las bajas laborales que ellos mismos han venido incrementando. También es pertinente precisar que los factores psicosociales son condiciones presentes en situaciones laborales relacionadas con la organización del trabajo, el tipo de puesto, la realización de la tarea, e incluso con el entorno, que afectan al desarrollo del trabajo y la salud de los trabajadores; y que los términos "organización del trabajo" y "factores organizativos" son intercambiables en el contexto laboral con "factores psicosociales" para señalar las condiciones de trabajo que conducen al estrés (Gil-Monte, 2009; Cabello et al., 2005).

Es claro, además, que los factores de riesgos psicosociales son los que más pueden alterar o favorecer el crecimiento del individuo, tanto dentro como fuera de la organización; por lo cual es importante que tengan en cuenta que cuando se fomenta el desarrollo del individuo mejora la calidad de vida 
laboral y social de la persona, mientras los factores desfavorables perjudican la salud y el bienestar.

Según Gil-Monte (2012), los riesgos psicosociales que origina la actividad laboral pueden ocasionarse por el deterioro o disfunción de los siguientes procesos:

a) Las características de la tarea: identificadas en la cantidad de trabajo, desarrollo de aptitudes, monotonía o repetitividad, ritmo de trabajo, falta de autonomía, etc.

b) Las características de la organización: en las que cuentan variables estructurales, definición de competencias, estructura jerárquica, canales de comunicación e información, relaciones interpersonales, procesos de socialización y desarrollo de la carrera, estilo de liderazgo, tamaño, etc.

c) Las características del empleo: diseño del lugar de trabajo, salario, estabilidad en el empleo y condiciones físicas.

d) La organización del tiempo de trabajo: duración y tipo de jornada, pausas de trabajo, trabajo en festivos, trabajo a turnos y nocturno, etc.

Los riesgos psicosociales son de carácter cualitativo y descriptivos, y se encuentran inmersos en la estructura organizacional donde se evalúa el clima organizacional y el liderazgo, entre otras características que nos pueden predecir la aparición de problemas psicológicos asociados a las condiciones de trabajo que afectan la salud y el bienestar de los trabajadores. Cuando las condiciones laborales actúan como un importante factor de riesgo psicosocial, provocan tensión, estrés o Burnout, entre otros (Maslach \& Jackson, 1986; Bernat \& Caso, 2010).

De acuerdo con los autores citados, la disfunción de los factores organizacionales en relación con el factor psicosocial provoca respuestas no adaptativas, causantes de estrés, así como respuestas psicofisiológicas adversas, que afectan la salud física a través de mecanismos psicofisiológicos activados por el estrés.

Entonces, aunque desde los inicios de la humanidad, el trabajo ha resultado importante en la determinación del crecimiento integral, pues lleva a las personas a alcanzar metas y objetivos dentro de una organización, este también puede afectar la salud física o psicológica, dependiendo del entorno y de los factores en los cuales el ser humano se desenvuelva para ejecutar su tarea. De ahí que, a nivel mundial, estos riesgos tienen una mayor probabilidad de causar daño a la salud del trabajador, tal es el caso del estrés laboral, 
el cual es considerado como una pandemia de la actualidad (Moreno, 2011; Benavides et al., 2002).

Por otra parte, cuando se adoptan medidas preventivas tempranas en materia del riesgo psicosocial se puede obtener una reducción considerable de los costos económicos y sociales tanto para el trabajador como para el empleador y los empresarios.

En Colombia, la primera encuesta nacional sobre condiciones de salud y trabajo, que fue realizada en el año 2007, mostró que el $30 \%$ de los trabajadores encuestados manifestó sufrir estrés laboral; mientras que en la segunda encuesta, realizada entre los periodos 2009 y 2012, se produjo un notable incremento, dado que el $43 \%$ de los trabajadores encuestados sufrían este tipo de riesgo psicosocial (Gutiérrez \& Viloria, 2014).

Según los datos aportados en el 2007, los factores que causan el riesgo psicosocial son variados: la carga laboral, las presiones económicas (el endeudamiento del hogar), la falta de reconocimiento a la labor realizada, la exigencia o la necesidad de realizar horas extras, la imposibilidad de cumplir con las exigencias operativas que demanda el puesto de trabajo y las expectativas inalcanzables personales o laborales, entre otras. Lo anterior hace que irremediablemente el trabajador sufra las consecuencias del riesgo psicosocial, afectando no solo la individualidad sino el entorno.

La principal consecuencia del riesgo psicosocial es el estrés, cuyos síntomas más frecuentes comprenden desde la irritabilidad hasta la depresión y por lo general están acompañados de agotamiento físico y mental. De esta manera, el estrés incide directamente en el desempeño del trabajador, ocasionando una baja productividad y afectando el confort laboral. $Y$ es que una persona sometida a una tensión muy alta es incapaz de tomar decisiones correctas y disminuye notoriamente su creatividad, pues la mente es incapaz de encontrar las múltiples soluciones que se requieren para un desempeño exitoso. Güilgüiruca et al., (2015) plantean al respecto que una empresa con muchas personas estresadas presenta generalmente un mal clima laboral.

Hoy en día, cuando un individuo pasa la mayor parte de su tiempo en el lugar de trabajo, es indispensable que exista un ambiente y clima laboral que incentive la motivación y aporte el bienestar que necesita.

Como se ha mencionado anteriormente, los trabajadores agobiados tienden a somatizar de diversas maneras el estrés, lo que hace que a menudo 
presenten síntomas fisiológicos como: tensión muscular, disminución del sistema inmunológico, problemas cardiovasculares, alergias, fibromialgias, dolor lumbar, problemas de la piel, migrañas, dificultades respiratorias, infertilidad transitoria y problemas gastrointestinales. Además, aparecen síntomas emocionales: ansiedad, depresión, irritabilidad, alteraciones del sueño, aburrimiento, frustración, aislamiento, dificultades de concentración, indecisión, pérdida de la memoria y síntomas conductuales tales como agresividad, abuso de alcohol y drogas, trastornos alimenticios, conflictos interpersonales, trastornos sexuales, ausentismo laboral, disminución de la producción y una mayor probabilidad de sufrir accidentes (Güilgüiruca et al., 2015; Cox \& Rial-González, 2000).

Teniendo en cuenta todo lo expuesto, es importante que los empleadores comprendan que una intervención tardía al respecto conlleva una reducción de la productividad y el descenso de la calidad de vida de aquellos que los sufren, pudiendo incluso ser motivo de baja laboral.

El ser humano es complejo por naturaleza, y representa un reto para el futuro de las empresas, tomando en cuenta que el hombre (su fuerza laboral) es el recurso más importante. En este marco, la necesidad de incorporar procesos que comprendan y atiendan todas las dimensiones humanas y laborales de las personas que integran cada empresa y la importancia dada a la relación organización-individuos permite identificar la influencia de los factores psicosociales y organizacionales en la productividad, así como trabajar en estrategias que permitan el mejoramiento continuo de los procesos y la identificación y eliminación o control de los factores de riesgos psicosociales.

Identificar estos factores repercute de forma positiva en el mejoramiento de la productividad (Hernández, 2011), representando un elemento estratégico para diseñar las acciones o planes para este fin.

Por otra parte, hoy en día las organizaciones persiguen el desarrollo organizacional orientado hacia el talento humano. Es decir, se busca reconocer al trabajador siempre como el punto de partida en el mejoramiento continuo y en los cambios que se supone mejoran los procesos organizativos y productivos que deben darse dentro de las estructuras organizacionales.

Existen elementos diferenciales de los factores psicosociales de riesgo que no deben obviar el interés básico de la SST:

1. Se extienden en el espacio y el tiempo 
2. Dificultan la objetivación

3. Afectan a los otros riesgos

4. Tienen escasa cobertura legal

5. Están moderados por otros factores

6. Dificultan la intervención

7. Afectan a los derechos fundamentales del trabajador

8. Tienen efectos globales sobre la salud del trabajador

9. Afectan la salud mental de los trabajadores

10. Tienen formas de cobertura legal

Los riesgos psicosociales se caracterizan por la no localización. Es decir, una cultura organizacional, un estilo de liderazgo o un clima psicosocial no se ubican en ningún lugar ni se pueden precisar en un momento especial (Rick y Briner, 2000). Esta situación, aunada a otros factores laborales como la carga de trabajo, los roles o la capacidad de control, que tampoco tienen un lugar y un momento propios, sino que son elementos del propio puesto de trabajo o la tarea, revelan la importancia de mantener los controles de vigilancia adecuados.

Como bien se ha mencionado a lo largo de este artículo, una de las principales si no la principal manifestación de los riesgos psicosociales es el estrés agudo. De hecho, sus efectos principales, como la violencia, el acoso laboral o el acoso sexual afectan directamente el funcionamiento de la persona que trabaja. Además, tras él, se pueden presentar otras formas de riesgos psicosociales como la inseguridad laboral o el síndrome de Burnout o desgaste profesional, todas en sí formas de estrés crónico que afectan a la totalidad de la organización, e incluso pueden llevar a la somatización de estas conductas, manifestándose en enfermedades que afectan directamente la productividad.

Como se ha establecido a lo largo de esta propuesta reflexiva, es importante identificar los factores psicosociales y organizacionales que afectan la productividad y el bienestar laboral, de manera que se puedan desarrollar las estrategias adecuadas para contrarrestar sus efectos negativos, y generar planes de acción encaminados a satisfacer las necesidades y objetivos organizacionales y personales de los empleados. En la medida en que las personas identifiquen los riesgos, se sentirán acompañadas en sus procesos y sentirán que son parte activa de los procesos y de la cultura de la organización, todo lo cual redundará en una mayor productividad, acompañada de un alto sentido de pertenencia hacia la empresa. 
La estrategia preventiva se ha consolidado como el primer paso en la promoción del bienestar en el trabajo, las organizaciones han comprendido que si existe un confort físico, moral y social se alcanzarán más fácilmente los objetivos. En tal sentido el enfoque de la política de calidad y seguridad y salud en el trabajo debe incluir la prevención de los riesgos sociales: el estrés, el acoso en el trabajo, la depresión, la ansiedad y los riesgos asociados a la dependencia del alcohol, las drogas o los medicamentos.

Así las cosas, cuando las organizaciones son conscientes de la necesidad y del deber de cuidar al talento humano integran en sus actividades de prevención la evaluación e intervención en materia de riesgos psicosociales, adaptando el actual marco normativo para la integración de los factores de riesgos psicosociales a los que se encuentran expuestos, en particular los que se destaquen dentro de la organización.

Los datos actuales indican que la ansiedad y la depresión, además de otros indicadores de mala salud mental están asociadas a la exposición a los riesgos psicosociales (Cox, Griffiths y Leka, 2005; Cox y Rial-González, 2000). Además, cuando un trabajador se encuentra expuesto a riesgos psicosociales sin que existan las medidas pertinentes para evitar o controlar sus consecuencias, se constituye una violación importante de las expectativas laborales básicas, no se concreta entonces el cumplimiento al derecho de un trabajo digno, por lo que las consecuencias de la exposición a estos factores de riesgos pueden generar alteraciones de carácter legal en los procesos organizacionales.

Asimismo, la aparición de síntomas como la depresión, conductas parasuicidas o inclusive el suicidio no solo afectan al empleado, sino a su entorno familiar (Moreno \& Mantilla, 2016). A lo que cabe agregar el trastorno de estrés postraumático, que se ha hecho tan frecuente en contextos laborales que ha sido sugerido como una enfermedad profesional.

Plantear enfoques adecuados dentro de las organizaciones para abordar la perspectiva y los paradigmas actuales alrededor del tema que hemos discutido en este artículo, puede suponer el éxito de las medidas adoptadas en la prevención y el tratamiento de los riesgos psicosociales, así como también de las políticas que debe crear cada organización en torno al tema. Actualmente, la legislación, así como la jurisprudencia colombiana y los bloques de constitucionalidad que tienen arraigo en la normatividad internacional tratan el tema con destacada importancia y brindan soluciones eficientes ante la vulnerabilidad de la población trabajadora. Por tanto, es 
obligación de las organizaciones garantizar en todo momento y de todas las maneras posibles la salud física y mental de sus trabajadores.

\section{CONCLUSIONES}

Para llevar a cabo una prevención eficaz y la adecuada intervención frente a las tendencias y nuevas orientaciones que direccionan los aspectos relacionados con los riesgos psicosociales, debe existir un compromiso total y absoluto desde las alta dirección de las organizaciones, así como recursos específicos y suficientes. Solo así se podrán identificar, analizar y valorar las probables causas, y de acuerdo a estos análisis surgirán soluciones efectivas de control y eliminación de los riesgos de acuerdo con el riesgo y con cada caso.

Sin embargo, son más recomendables las medidas de carácter general y organizativas que las particulares, debido a que cuando se realiza la detección temprana de los riesgos, y se ejecutan actividades de mitigación o eliminación, los resultados tienden a ser más positivos; es así como las actividades de prevención individual han de ser posteriores o complementarias a las actividades de prevención de carácter global. Lo anterior ha sido contemplado por autores como Gil-Monte (2009), no solo para lograr mayor sostenibilidad en el tiempo sino también un ambiente óptimo para su acogida. Así se puede garantizar que las inversiones de tiempo y recursos cumplan su objetivo principal, que consiste en aminorar el impacto del riesgo psicosocial en el trabajador.

Estos tipos de intervenciones deben encaminarse a facilitar la autonomía, el desarrollo y la satisfacción en el trabajo, de modo que se promuevan las actitudes de cooperación. En concreto, deben emplearse medidas de prevención como: Modificar la organización del trabajo, enriquecer las tareas, permitir la autonomía en su desarrollo, rotar tareas y puestos; mejorar el medioambiente de trabajo, controlar riesgos; propiciar espacios de trabajos adecuados; realizar un manejo razonable del tiempo de trabajo estableciendo pausas y descansos adecuados; suministrar información asertiva sobre los procesos y las tareas a realizar; posibilitar la comunicación formal e informal entre los trabajadores y superiores; favorecer la participación de los trabajadores, etc. Esto coincide con informes e investigaciones como la de Gutiérrez y Viloria (2014), quienes sugieren que cuando se renuevan y reorganizan los escenarios laborales, se pueden lograr dinámicas e interacciones realmente ventajosas para la mejora de los entornos. Esto último no solo es bueno 
porque involucra todos los actores del entorno o ámbito laboral, sino porque también promueve la identificación de nuevas oportunidades.

Nota de autores: Articulo avance de investigación de un proyecto desarrollado entre las universidades de La Guajira, Atlántico y Corporación Universitaria Minuto de Dios, "Tendencias del riesgo ocupacional en el contexto educativo".

\section{REFERENCIAS}

Benavides, F., Gimeno, D., Benach, J., Martínez, J. M., Jarque, S. \& Berra, A. (2002). Descripción de los factores de riesgo psicosocial en cuatro empresas. Gaceta Sanitaria, 16(3), 222-229. https://doi.org/10.1016/S0213-9111(02)71665-8

Bernat, A. \& Caso, C. (2010). Procedimiento en materia de prevención, resolución y seguimiento de las situaciones de conflicto y acoso psicológico en el entorno laboral. Medicina y Seguridad del Trabajo, 56(1), 124-131. Disponible en https:// www.educacion.navarra.es/documents/57308/57782/Procedimiento_violencia_y_acoso.pdf/2a4998bd-d475-4ac2-9a3b-0e6c4229d847

Cabello, R., Fernández-Berrocal, P., Ruiz, D. \& Extremera, N., (2005). Influencia de la Inteligencia emocional y estilos de respuesta en factores de riesgo psicosocial en profesores de educación especial. 6o Congreso Virtual de Psiquiatría. Disponible en https://psiquiatria.com/psicologia/influencia-de-la-inteligencia-emocional-y-estilos-de-respuesta-en-factores-de-riesgo-psicosocial-en-profesores-de-educacion-especial/

Cox, T. (2004). Work-related stress, risk management and management standards. Work \& Stress, 18(3), 89-90. Disponible en http://www.hse.gov.uk/stress/ standards/

Cox, T., Griffiths, A. \& Leka., S. (2005). Work organization and work related stress. In K. Gardiner. \& J. M. Harrington (Ed.), Occupational Hygiene. Oxford: Blackwell.

Cox, T. \& Rial-González, E. (2000). Risk management, psychosocial hazards and work stress. In J. Rantanen \& S. Lehtinen (Ed.), Psychological Stress at Work. Helsinki: Finnish Institute of Occupational Health.

Gil-Monte, P. R. (2009). Algunas razones para considerar los riesgos psicosociales en el trabajo y sus consecuencias en la salud pública. Rev. Esp. Salud Pública 2009; 83, 169-173. Disponible en http://scielo.isciii.es/scielo.php?script=sci_arttext\&pid=S1135-57272009000200003

Gil-Monte, P. R. (2012). Riesgos psicosociales en el trabajo y salud ocupacional. Rev. Perú, Med. Exp. Salud Publica, 29(2).

Güilgüiruca, M., Meza, K., Góngora, R. \& Moya, C. (2015). Factores de riesgo psicosocial y estrés percibido en trabajadores de una empresa eléctrica en Chile. Med. segur. Trab, 61(238). http://scielo.isciii.es/scielo.php?script=sci_arttext\&pi$d=S 0465-546 \times 2015000100006$

Gutierrez, A. \& Viloria, J. (2014). Riesgos psicosociales y estrés en el ambiente laboral. Revista Salud Uninorte, 30(1). http://dx.doi.org/10.14482/sun.30.1.4340

Hernández, H. (2011). La gestión empresarial, un enfoque del siglo XX, desde las teorías administrativas científica, funcional, burocrática y de relaciones humanas. Revista Escenarios, 9(1), 38-51. Disponible en https://dialnet.unirioja.es/servlet/ articulo?codigo $=3875234$

Maslach, C. \& Jackson, S. E. (1986). Maslach Burnout Inventory 20 Edición. Palo Alto, California: Consulting Psychologists Press. 
Moreno, B. (2011). Factores y riesgos laborales psicosociales: conceptualización, historia y cambios actuales. Med Segur Trab (Internet) 2011, 57. Suplemento 1, 1-262. Disponible en http://scielo.isciii.es/scielo.php?script=sci_arttext\&pi$d=S 0465-546 \times 2011000500002$

Moreno, G. \& Mantilla, J. (2016). Una revisión del concepto observatorio social: hacia una comprensión de sus objetivos, alcances, métodos y finalidades. Psicogente, 19(36), 347-359. https://doi.org/10.17081/psico.19.36.1303

OIT/OMS. (1984). Factores Psicosociales en el Trabajo: Naturaleza, incidencia y prevención. Disponible en: http://www.ergonomia.cl/eee/Noticias_anteriores/ Entradas/2013/11/1_OIT_OMS_Factores_Psicosociales_en_el_Trabajo.html

Organización Mundial de la Salud (OMS). (2010). Entornos Laborales Saludables: Fundamentos y Modelo de la OMS Contextualización, Prácticas y Literatura de Apoyo. Disponible en: http://www.who.int/occupational_health/evelyn_hwp_ spanish.pdf

Rick, J. \& Briner, R. B. (2000). Psychosocial Risk Assessment: Problems and Prospects. Occupational Medicine, 50(5), 310-314. Disponible en https://www.ncbi.nlm.nih. gov/pubmed/10975126

Singh, A.P. (1998). Supervision and organizational effectiveness: Role conflict as a moderator. Journal of the Indian Academy of Applied Psychology, 24(1-2), 19-25. Disponible en http://medind.nic.in/imvw/imvw7911.html autor o el licenciante.

\section{(cc) BY}

